\title{
Kreditgenossenschaften und ihr ausgleichender Beitrag im Markt
}

Rezension zu Georg Scheumann (2017): Die Abkehr von der Genossenschaftsidee - Werden die Mitglieder der Volks- und Raiffeisenbanken verraten und verkauft? Bullay: union design group i.G.

In wirtschafts- und unternehmensethischer Hinsicht erweisen sich die Rechtsformen der eingetragenen Genossenschaft (eG), aber auch des Vereins (e.V.), der Stiftung oder des Versicherungsvereins auf Gegenseitigkeit (VVaG) als »anders als die anderen « (Brazda et al. 2006). Dies geht im Kern darauf zurück, dass Unternehmen in diesen Rechtsformen stärker sach- und personenorientiert angelegt sind als gewinnorientiert. Im Mittelpunkt sollen die Träger bzw. ein Sachziel als (Stiftungs-)Zweck stehen.

Bei Genossenschaften ist dies die Förderung der Eigentümer (Mitglieder) durch gute und günstige Leistungen, wie sie so am Markt momentan nicht, vielleicht sogar nie, nicht mehr oder noch nicht zu bekommen sind. Die Genossenschaft schließt diese Lücke und behebt eine wirtschaftliche Mangelsituation. Das Vorhandensein von Genossenschaften im Markt erweist sich, auch in überraschend eintretenden Marktkrisen wie der 2007 einsetzenden Finanzmarktkrise und im historischen Maßstab, als äußerst stabilisierend. Damit erhalten Genossenschaften zu jeder Zeit das Angebot und die Versorgung mit ihren Leistungen für Mitglieder und Kunden aufrecht.

Anfänglich waren sogar Märkte selbst als Genossenschaft organisiert. Mit dieser Perspektive stellt sich ein Markt als soziale Koordinationsform dar, die durchaus einen sozialen Beitrag zu erbringen vermag.

In ähnlicher Weise zeichnet Kreditinstitute, vor allem Kreditgenossenschaften und Sparkassen, eine soziale Funktion aus. Doch sie werden schon seit längerem in Richtung rein erwerbswirtschaftlicher Unternehmen entwickelt. Dazu passt es, dass aktuell die Rechtsform der eG in der Kreditwirtschaft unter dem aufsichtsrechtlichen Anpassungsdruck in Richtung des Modells eines Kreditinstitutes in der Rechtsform der AG steht.

Auf europäischer Ebene scheinen Verständnis für und Wissen um die Besonderheiten von Kreditgenossenschaften nur gering ausgeprägt zu sein. Vor diesem Hintergrund sind alle Publikationen, die dies zu beheben versuchen, schon wertvoll an sich.

Das Buch von Georg Scheumann, einem früheren Vorstandsmitglied einer Kreditgenossenschaft, die eine vom Prüfungsverband empfohlene Fusion so nicht eingehen wollte, widmet sich der grundlegenden Frage, inwieweit die heute noch in

* Dr. Holger Blisse, Wien/Österreich, ist Wirtschafts- und Sozialwissenschaftler und auf kreditwirtschaftliche, genossenschaftliche und sozial-politische Themen spezialisiert, EMail: holger.blisse@gmx.at. 
Deutschland bestehenden Kreditgenossenschaften, Volksbanken und Raiffeisenbanken trotz ihrer rechtlichen Eigenständigkeit frei in ihren Entscheidungen und Handlungen sowie ihrem Gründungsgedanken treu geblieben sind und ihre Identität wahren (vgl. Bonus 1994). Scheumann sieht, wie sich Management und Mitglieder voneinander entfernt haben und damit die Vorteile der eG nicht mehr den Mitgliedern (und Kunden) zugutekommen, sondern andere Interessen, auch diejenigen der (Prüfungs-) Verbände, in den Vordergrund rücken.

Man wird sich nicht allen seinen Argumenten anschließen, z.B. zum Geschäft mit Nichtmitgliedern (vgl. 140f.), die man durchaus auch als potenzielle Mitglieder interpretieren könnte. Dennoch bietet das Werk, indem es die Funktionsweise der Genossenschaft und ihrer Organe mit Bezug zum Genossenschaftsgesetz erklärt und um Kommentare aus der Wissenschaft ergänzt, einen guten und durchaus trotz Betroffenheit des Autors Sachlichkeit wahrenden Überblick. Es informiert über eine gesetzeskonforme Funktionsweise einer Kreditgenossenschaft und leistet damit zugleich einen wertvollen Beitrag, die Diskussion um den Erhalt und die Fortentwicklung der genossenschaftlichen Substanz innerhalb einer stärker werdenden und zur Konzentration und damit zu steigender Macht und höheren Preisen neigenden Wettbewerbswirtschaft zu verbreitern.

Hinsichtlich der Rolle der Prüfungsverbände und der Pflichtmitgliedschaft in einem Prüfungsverband mag man durchaus anderer Auffassung sein. Dennoch sensibilisiert der Autor für ein sich verstärkendes Kräfteungleichgewicht nicht nur zugunsten des Managements, sondern gerade auch der Interessen-/Prüfungsverbände und damit zu Ungunsten der originären Eigentümer. Dies erweist sich gerade deshalb als problematisch, da in den Kreditgenossenschaften und in ihrem Verbund über die Jahrzehnte ein Vermögen angewachsen ist, das aktuell in den Blick verschiedener Interessen zu geraten scheint.

Das Vermögen einer eG ist dem Zugriff der Mitglieder während und bei Beendigung ihrer Mitgliedschaft sehr weitgehend entzogen. Dies führt immer wieder zu Fragen der Verteilung(sgerechtigkeit) und Zurechenbarkeit (vgl. z.B. van Husen 1998; Beuthien/Klappstein 2018). In gewisser Weise ist dieses Vermögen keiner Mitgliedergeneration allein direkt zurechenbar, wenn es durch den in der genossenschaftlichen Rechtsform angelegten freiwilligen Verzicht früherer Generationen auf Ausschüttungen gewachsen ist. Dem Vorstand einer Genossenschaft kommt beim Erhalt dieses Vermögens eine besondere Treuefunktion zu, die heute als gefährdet angesehen werden kann. Kritisch sieht Scheumann auch die zunehmende Dotierung eines Fonds für allgemeine Bankrisiken (vgl. 87-91), der dem Zugriff der Mitglieder grundsätzlich entzogen ist und die Risikoneigung des Managements in einem am Markt orientierten Geschäftsumfeld nicht unbedingt mindert. Da zudem das Vermögen innerhalb des kreditgenossenschaftlichen Verbundes beträchtlich gewachsen ist, sollte auch dieses einem besonderen Schutz und einer besonderen Treuepflicht unterliegen. Höchstens wäre noch eine mittelbare Beteiligung der Mitglieder an diesem Vermögen zu erwägen (vgl. Blisse 2017). Denn die Kreditgenossenschaften sind »stellvertretend für ihre Mitglieder « (Bonus 1990: 263) Eigentümer dieses Vermögens. 
Wenn sich die Vorstellungen auf europäischer Ebene jedoch durchsetzen, Kreditgenossenschaften - und Sparkassen - in Aktiengesellschaften zu überführen, ihr Vermögen damit in Aktien zu verbriefen und dem Zugriff des Marktes bis hin zu einem Aufkauf und einer Übernahme zu erschließen, dann ginge eine wichtige Institution, die nicht auf Gewinnmaximierung angelegt ist, mit ihrer Preise und Wettbewerb moderierenden Wirkung im Markt verloren.

Georg Scheumann ist zuzustimmen, dass alle Organe die ihnen zukommenden Funktionen wieder ausfüllen sollten. Hier stehen zweifelsfrei ebenso die Genossenschaftsverbände in der Pflicht, auch auf europäischer Ebene gegenzusteuern, anderenfalls ist die Gefahr groß, dass sich das kreditgenossenschaftliche System weiter zentralisiert bzw. tatsächlich auflöst. Die Banken würden fusioniert, in Aktiengesellschaften umgewandelt oder verkauft oder ihr Vermögen würde umgewidmet, jedenfalls gingen sie als nicht auf Gewinnmaximierung angelegte Banken verloren. Damit nähme das Angebot für Bankkundinnen und Bankkunden ab und würde teurer. Eine Situation, wie sie nach neuen Genossenschaften riefe - doch warum nicht diejenigen bewahren, die gerade heute noch diese Funktion ausüben? Mit dieser Leserichtung sei dem Buch eine weite Verbreitung gewünscht, die das Engagement jedes Mitgliedes für sseine eigene Kreditgenossenschaft, Volksbank und/oder Raiffeisenbank reaktiviert und deren Fortbestand als Genossenschaft und Kreditinstitut mit eigenem sozialen Wertesystem im regionalen oder sogar lokalen Maßstab nachhaltig sichert.

\section{Literaturverzeichnis}

Beuthien, V./Klappstein, V. (2018): Sind genossenschaftliche Rücklagen ein unteilbarer Fonds? - Zur Kapitalerhaltung und Überschussverwendung im Genossenschaftsrecht. Schriften zum Unternehmens- und Kapitalmarktrecht, Bd. 47, Tübingen: Mohr Siebeck.

Blisse, H. (2017): Genossenschaftliche Verbundfinanzierung als Alternative zur Kapitalmarktfinanzierung - Eine Option für Volksbanken und Raiffeisenbanken in Europa? Wien: Wissenschaftlicher Rat.

Bonus, H. (1990): Zur Eigentümerproblematik von Kreditgenossenschaften und Verbund Überarbeitete Fassung eines Referates auf der Bankleiter-Fachtagung des Genossenschaftsverbandes Niedersachsen e.V. am 27. April 1989 in Göttingen, in: Rheinisches Genossenschaftsblatt, o. Jg., 263-266, 305 (2. Teil).

Bonus, H. (1994): Das Selbstverständnis moderner Genossenschaften - Rückbindung von Kreditgenossenschaften an ihre Mitglieder, Tübingen: Mohr (Siebeck).

Brazda, J./Kramer, J. W./Laurinkari, J./Schediwy, R. (2006): Anders als die Anderen - Eine unbefangene Annäherung an Genossenschaften, Sozialwirtschaft und Dritten Sektor, Bremen: Salzwasser.

Husen, R. van (1998): Wem gehört das Genossenschaftsvermögen? - Ein Beitrag zur Reform des Genossenschaftsrechts. Studien zum Unternehmens- und Wirtschaftsrecht, Bd. 7, Wien: Verlag Österreich. 